Ann. Zootech., I974, 23 (3), 30I-3I2.

\title{
COMPOSITION DU JUS DE RUMEN CHEZ LE MOUTON RECEVANT A VOLONTÉ DU MAÏS FOURRAGE SUR PIED OU ENSILÉ
}

\author{
J. ANDRIEU et C. DEMARQUILLY \\ avec la collaboration technique de J.-M. BoIssau, H. BousqueT \\ Jacqueline JAMOT, Marie JAILLER et L. L'Hotelier \\ Laboratoire des Aliments, \\ Centre de Recherches de Clermont-Ferrand, I. N.R.A., \\ Theix, 63110 Beaumont
}

\section{RÉSUMÉ}

Le $\mathrm{pH}$ et la concentration en acides gras volatils totaux (AGVT) du jus de rumen chez le mouton recevant I9 échantillons de maïs vert ont été en moyenne de 6,3 $\pm 0, \mathrm{I}$ et de $85,0 \pm \mathbf{1 2 , 3}$ mmoles/litre de jus. Quant aux proportions molaires (p. roo) d'acides acétique, propionique, butyrique et d'acides ayant une longueur de chaîne égale ou supérieure à celle de l'acide valérianique, elles ont été en moyenne respectivement de $5.5,2 \pm 3,0 ; 29,6 \pm 3,8$; I3, I $\pm 2,6$ et $2,1 \pm 0,6$.

Si l'influence du stade de végétation et de ses principaux critères a été nulle sur le pH et très faible sur la composition du mélange d'acides gras volatils ( $\mathrm{AGV}$ ), elle a été par contre importante sur la concentration en AGVT : cette dernière a été d'autant plus faible que le stade de végétation de la plante était plus tardif.

La quantité de matière organique digestible ingérée par le mouton n'a eu une influence que sur les proportions molaires des différents AGV : la proportion d'acide propionique a augmenté au détriment des proportions d'acides acétique et butyrique quand la quantité de matière organique digestible ingérée a augmenté.

La conservation par l'ensilage ne s'est traduite que par une augmentation significative de la proportion d'acide butyrique au détriment de la proportion d'acide propionique.

Le $\mathrm{pH}$ et la concentration en AGVT du jus de rumen chez le mouton recevant I 4 ensilages de plantes entières sans additif ont été en moyenne respectivement de $6,2 \pm 0,2$ et $78,2 \pm 7,4$ mmoles/litre. Les proportions molaires (p. roo) d'acides acétique, propionique, butyrique et d'acide ayant une longueur de chaîne supérieure ou égale à celle de l'acide valérianique ont été en moyenne respectivement de $53,3 \pm 4,9 ; 27,3 \pm 4,0 ; 16,7 \pm 2,8$ et $2,7 \pm \mathrm{I}, \mathrm{I}$.

Les caractéristiques du jus de rumen chez le mouton recevant ces if ensilages de plantes entières ont été indépendantes des principaux critères du stade de récolte et des quantités de matière organique digestible, d'amidon ou d'acides préformés dans l'ensilage, ingérées par le mouton.

L'incorporation à la mise en silo d'un mélange d'urée et de minéraux a entraîné une augmentation importante de la quantité de matière organique digestible ingérée par le mouton et de la concentration en AGVT du jus de rumen. Les autres caractéristiques du jus de rumen ont été peu modifiées.

Les proportions d'acide acétique dans le jus de rumen ont été en moyenne de 57,$2 ; 53,2$ et $6 \mathrm{I}, 5 \mathrm{p}$. Ioo lorsque le mouton a reçu respectivement les ensilages de tiges + feuilles, de plantes 
entières et d'épis correspondant aux mêmes mais. Cependant les ensilages d'épis étaient distribués avec $200 \mathrm{~g}$ de foin par jour.

Les principaux résultats obtenus sont discutés en essayant d'en tirer un certain nombre de conclusions d'ordre zootechnique.

\section{INTRODUCTION}

Il est maintenant bien établi que le ruminant tire la plus grande partie de l'énergie qui lui est nécessaire des acides gras volatils issus de la fermentation des aliments dans le rumen. La composition du mélange des acides gras volatils produits dans le rumen à partir d'un aliment donné permet de caractériser la valeur de cet aliment pour la production de viande ou la production laitière. L'influence du type de ration sur la composition du jus de rumen a fait l'objet de nombreux travaux (voir par exemple BALCH et Rowland, I957 ; BATH et Rook, I963). Cependant, parmi les rations étudiées, un petit nombre était à base d'ensilage de maïs et aucune, du moins à notre connaissance, ne contenait du maïs sur pied. C'est pourquoi, dans le cadre plus général de l'étude de la valeur alimentaire chez le mouton du mais fourrage (DEMARQUILIY, I969; ANDRIEU et DEMARQUILLY, I974 $a$ et $b$ ), nous avons étudié quelle était l'influence $d u$ stade de récolte de la plante de maïs distribuée en vert, de la conservation par l'ensilage, de l'addition d'urée et de minéraux à la mise en silo, et, enfin, de l'enrichissement en épis des ensilages sur la concentration du mélange d'acides gras volatils du jus de rumen et sur la composition de ce mélange.

\section{MATÉRIEL ET MÉTHODES}

Cinq mais, dont nous suivions l'évolution de la valeur alimentaire en vert sur des lots de 4 à 6 moutons, ont été distribués à un mouton supplémentaire muni d'une fistule du rumen. Les variétés étudiées, les lieux et les années d'études, ainsi que la durée des mesures sont indiqués dans le tableau I. Au total, cette étude a porté sur i9 échantillons dont les teneurs en matière sèche ont varié de i 6 à 33 p. roo, ce qui correspond à l'éventail des teneurs en matière sèche observé pour la plante de maïs entre la floraison et le début du stade vitreux.

\section{TABIEAU I}

Principales caractéristiques des mais étudiés en vert

\begin{tabular}{|c|c|c|c|c|c|}
\hline Année & Lieu & Variété & $\begin{array}{l}\text { Durée des } \\
\text { mesures }\end{array}$ & $\begin{array}{c}\text { Nombre } \\
\text { d'échantillons } \\
\text { étudićs }\end{array}$ & $\begin{array}{l}\text { Variations de la } \\
\text { teneur en matière } \\
\text { sèche de la plante } \\
\text { durant les mesures }\end{array}$ \\
\hline 1967 & Theix & $I N R A 200$ & $3 / 9-20 / 10$ & (;) & $16,2-21,9$ \\
\hline 1969 & Clermont-Id & $\begin{array}{ll}I N R A & 200 \\
I N R A & 258 \\
I N R A & 310 \\
I N R A & 400\end{array}$ & $\begin{array}{c}8 / 9-28 / 9 \\
8 / 9-28 / 9 \\
15 / 9-11 / 10 \\
15 / 9-4 / 10\end{array}$ & $\begin{array}{l}3 \\
3 \\
4 \\
4 \\
3\end{array}$ & $\begin{array}{l}27,6-32,9 \\
26,9-32,5 \\
23,4-31,4 \\
23,7-28,4\end{array}$ \\
\hline
\end{tabular}


Vingt ensilages de plantes entières, dont 8 correspondaient à des maïs étudiés en vert, 2 ensilages de tiges + feuilles et 8 ensilages d'épis (avec leurs spathes) ont été distribués également à un mouton supplémentaire muni d'une fistule du rumen. Afin de limiter la fréquence des indigestions observées dans le cas des ensilages d'épis, le mouton fistulisé, comme le lot de moutons utilisé pour les mesures de valeur alimentaire, ont reçu une quantité limitée de foin (zoo g/j/animal)

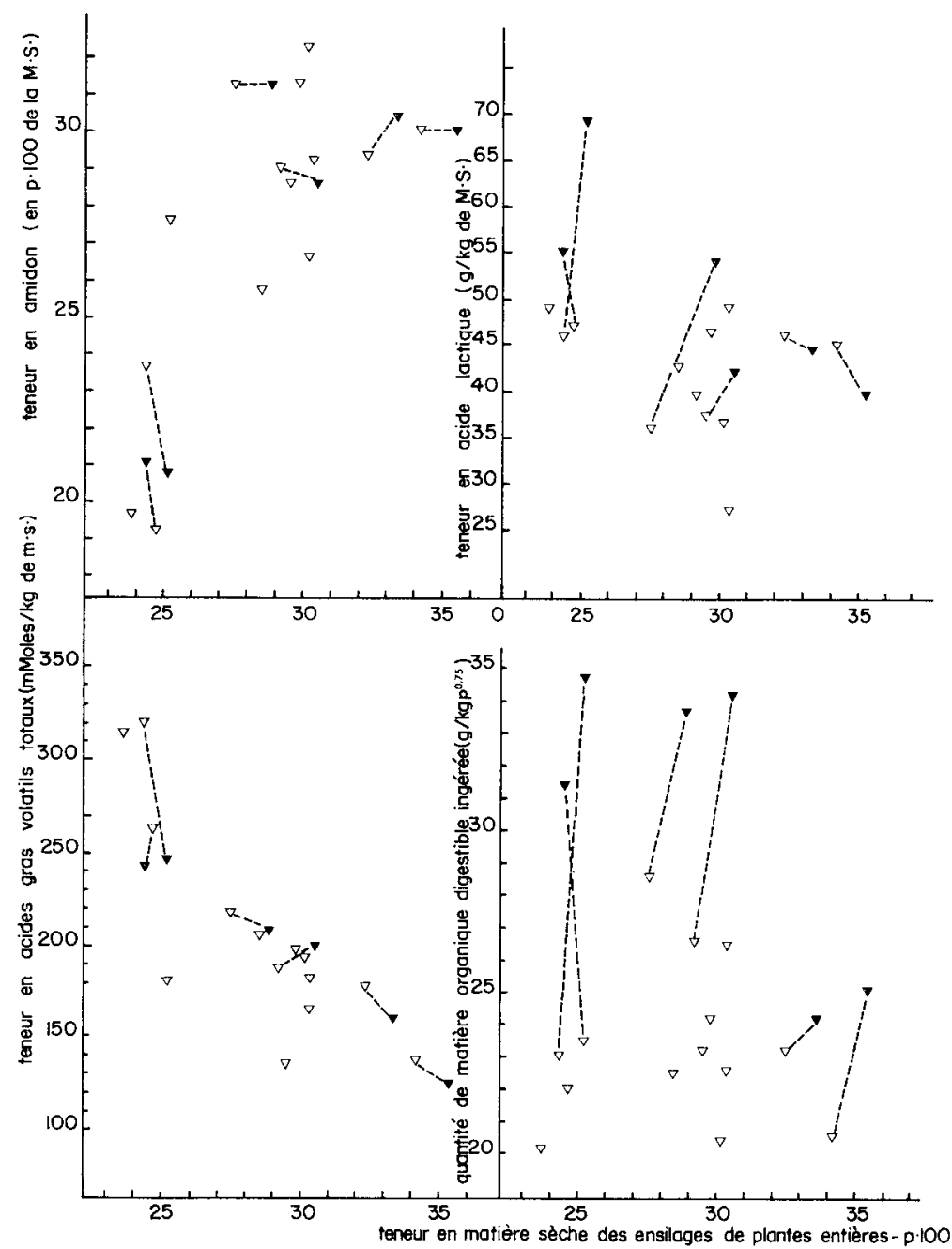

FIG. t. - Évolution de la composition et de la valeur alimentaire des ensilages de plantes entières en fonction de leur stade de récolte

$$
\text { Ensilage }\left\{\begin{array}{lll}
\nabla & \text { sans urée } \\
\nabla & \text { avec urée }
\end{array}\right.
$$

(les comparaisons d'ensilages avec ou sans urée sont reliées par un trait en pointillé)

environ $1 / 2$ heure avant la distribution du premier repas d'ensilage de la journée. Sur ces 30 ensilages, 6 ensilages de plantes entières et 4 ensilages d'épis contenaient un mélange d'urée et de minéraux ( $5 \mathrm{~g}$ d'urée et $5 \mathrm{~g}$ de minéraux $/ \mathrm{kg}$ de produit frais). Les principales caractéristiques des ensilages étudiés sont présentées dans la figure $\mathbf{I}$. 


\section{Mesures}

Le maïs fourrage a été distribué à volonté (Io p. Ioo de refus) soit en 3 repas par jour ( $7 \mathrm{~h} 30$, I 3 h 30 et $\mathrm{I} 7$ heures) dans le cas de la plante consommée en vert, soit en deux repas par jour $\left(8 \mathrm{~h} 3^{\circ}\right.$ et $\mathrm{I} 7 \mathrm{~h}$ ) dans le cas des ensilages. Nous avons prélevé du jus de rumen 2 heures après la distribution du premier repas de la journée et durant 2 jours consécutifs de chaque période de mesure de la valeur alimentaire. Le $\mathrm{pH}$ a été déterminé immédiatement et les échantillons ont été conservés à $-15^{\circ} \mathrm{C}$, après addition de ro $\mathrm{p}$. roo d'acide formique, jusqu'au moment de la détermination en chromatographie en phase gazeuse de leur teneur en acides gras volatils par la méthode de Rigaud et Journet (I970).

\section{RÉSULTATS}

\section{La plante de maïs consommée en vert}

La quantité de matière organique digestible ingérée par le mouton fistulisé a été légèrement inférieure à celle ingérée en moyenne par le lot de moutons $(32,2$ contre $35,7 \mathrm{~g}$ de $\mathrm{MOD} / \mathrm{kg} \mathrm{P}^{\mathbf{0}, 75}$ ).

Le $\mathrm{pH}$ du jus de rumen a été faible et surtout a peu varié : il a été en moyenne pour les I9 échantillons de $6,3 \pm 0, I$. La concentration en acides gras volatils totaux (AGVT) du jus de rumen a été par contre beaucoup plus variable puisqu'elle a été en moyenne de $85,0 \pm \mathrm{I} 2,3$ mmoles/litre de jus. Quant aux proportions molaires dans le jus de rumen, d'acides acétique, propionique, butyrique et d'acides ayant une longueur de chaîne égale ou supérieure à celle de l'acide valérianique, elles ont été en moyenne respectivement de $55,2 \pm 3,0$ p. I00; $29,6 \pm 3,8$ p. I00; $\mathrm{I3}, \mathrm{I} \pm 2,6$ p. I00; $2, \mathrm{I} \pm 0,6$ p. Iоo.

A la différence du $\mathrm{pH}$, qui a été indépendant du stade de végétation de la plante (fig. 2), la concentration en AGVT du jus de rumen ( $Y$ en mmoles/litre de jus) a diminué quand l'âge de la plante, et donc sa teneur en matière sèche ( $\mathrm{X}$ en $\mathrm{p}$. Ioo) augmentaient :

$$
\mathrm{Y}=-\mathrm{I}, 6 \mathrm{I} 7 * \mathrm{X}+\mathrm{I} 25,6 \mathrm{I} 8 \pm 8,99 \quad r=-0,705^{* * *} \quad n=\mathrm{I} 9
$$

Elle a également été liée significativement aux autres critères du stade de végétation de la plante comme la teneur en épis $\left(r=-0,69 \mathrm{I}^{* * *}\right)$, les teneurs en matières azotées totales $\left(r=+0,65 \mathrm{I}^{* *}\right)$ et en cellulose brute $\left(r=+0,553^{* *}\right)$. Les proportions des différents acides gras volatils (AGV) ont été indépendantes des principaux critères du stade de végétation de la plante comme, les teneurs en matière sèche (fig. 2) et en cellulose brute et la teneur en épis. Cependant, la proportion d'acide acétique a diminué faiblement mais significativement $(r=-0,53 ; \mathrm{P}<0,05)$ au profit de la proportion d'acide propionique quand la teneur en matières azotées a augmenté.

Si le $\mathrm{pH}$ et la concentration en AGVT ont été indépendants de la quantité de matière organique digestible ingérée, la composition du jus de rumen lui a été, par contre, étroitement liée : la proportion d'acide propionique a augmenté significativement $\left(r=+0,709^{* *}\right)$ au détriment des proportions d'acides acétique $(r=-0,634$ $\mathrm{P}<0,02)$ et butyrique quand la matière organique digestible ingérée a augmenté. En moyenne, une augmentation d'ingestion de Io $g$ de matière organique digestible

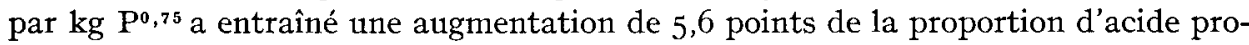
pionique au détriment de la somme des proportions d'acides acétique et butyrique qui a diminué de 5,4 points. 


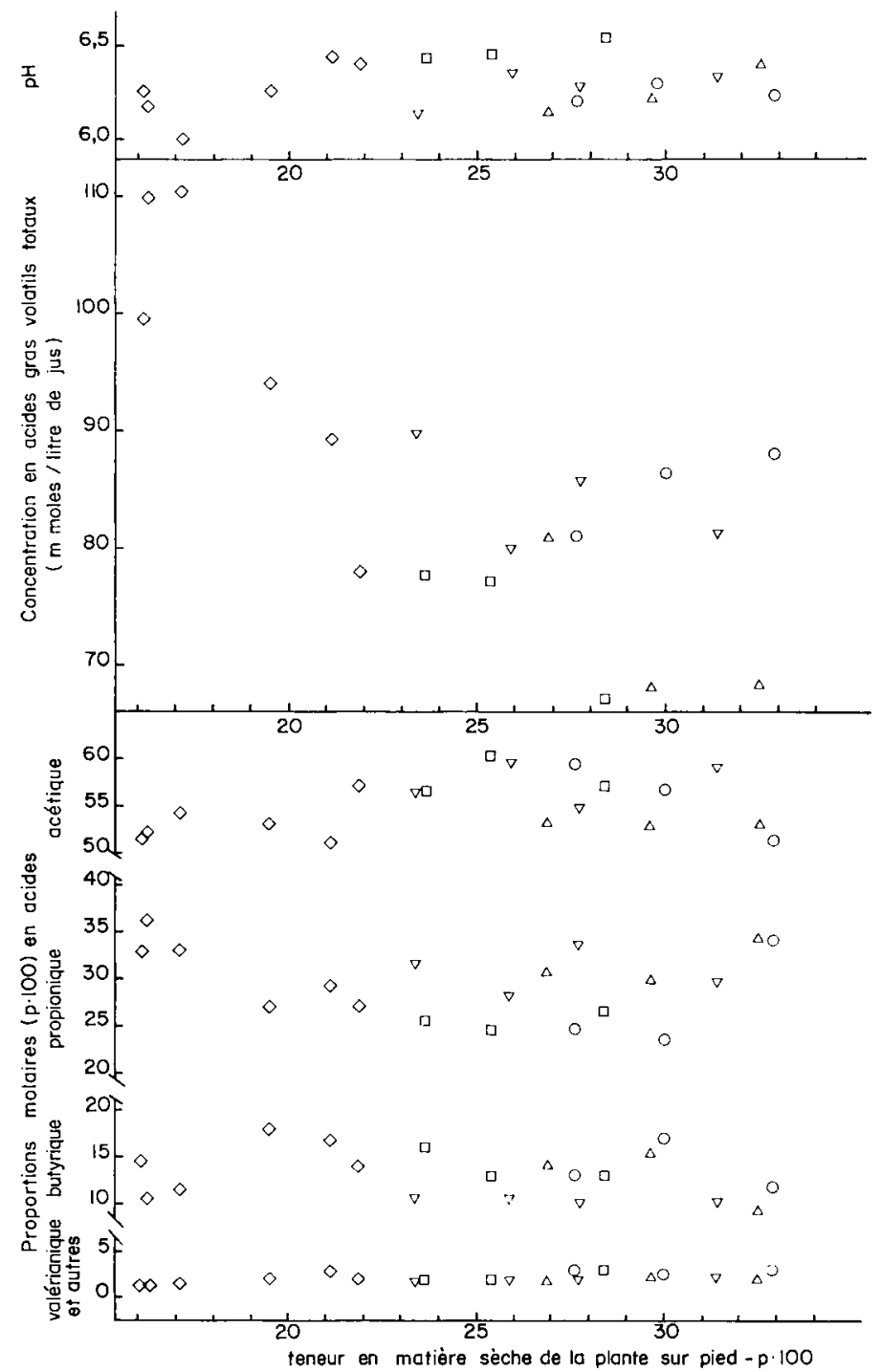

Frg. 2. - Influence de la teneur en matière sèche de la plante consommée en vert sur le pH, la concentration et la composition du jus de rumen
$\checkmark I N R A 200$, Theix I967
- INRA 200, Clermont-Ferrand 1969
$\triangle I N R A$ 258, Clermont-Ferrand 1969
$\nabla I N R A 310$, Clermont-Ferrand I969
¿ INRA 400, Clermont-Ferrand I 969

Ensilages de mais plante entière

Influence de la conservation.

La diminution d'ingestibilité entraînée par la conservation a été bien plus importante dans le cas du mouton fistulisé que dans le cas des moutons utilisés pour les mesures de valeur alimentaire ( 26 p. Ioo contre 6,8 p. IOo). La conservation par 1'ensi- 

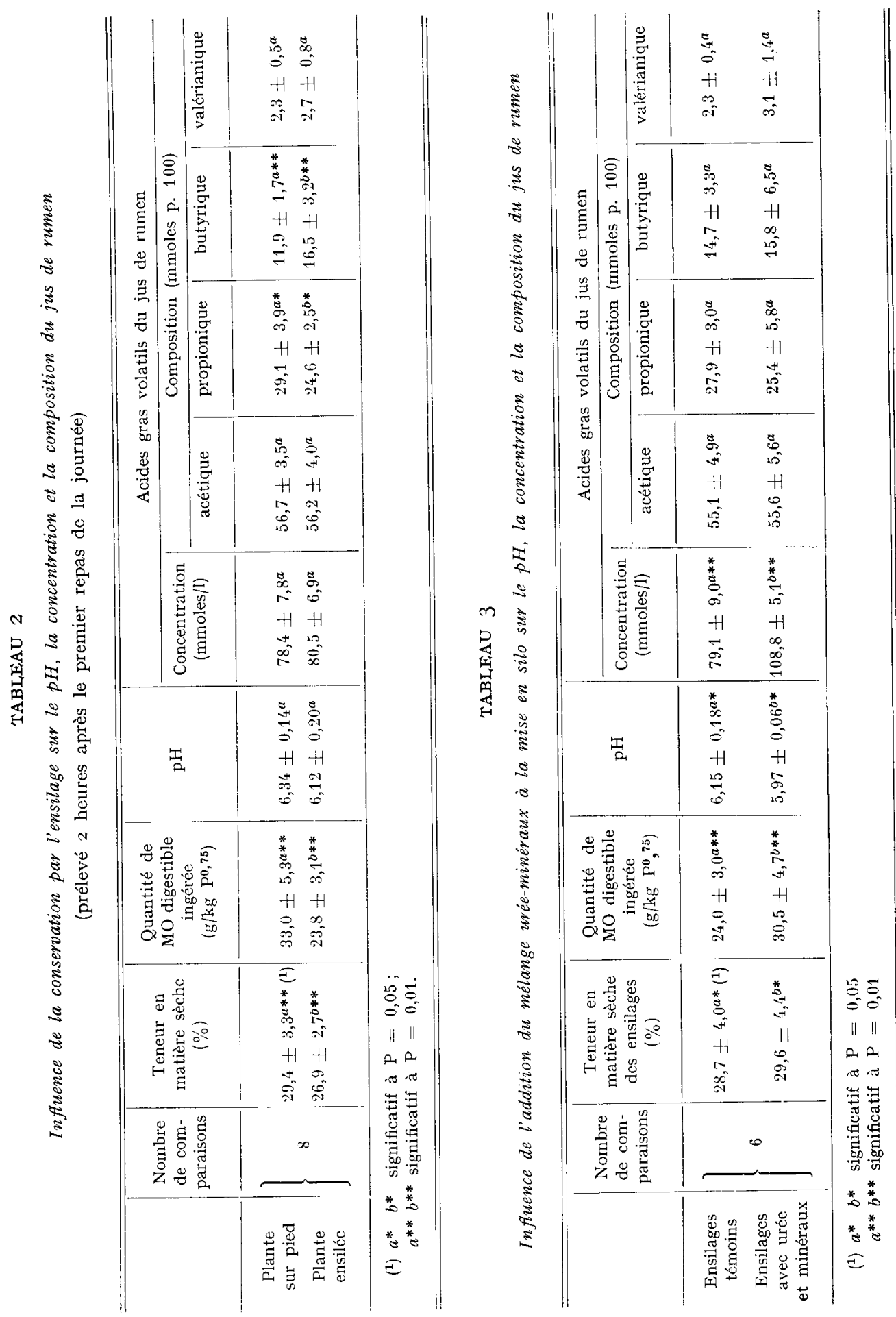
lage a entraîné des modifications faibles et non significatives du pH et de la concentration en AGV'T du jus de rumen (tabl. 2). De même, son influence sur la composition du mélange d'AGV cst restée faible ; elle s'est traduite cependant par une augmentation significative $(\mathrm{P}<0,0 \mathrm{I})$ de la proportion d'acide butyrique de $\mathrm{I} I, 9$ à $\mathrm{I} 6,5 \mathrm{p}$. IoO au détriment de la proportion d'acide propionique qui est passée de $29, \mathbf{I}$ à 24,6 p. Ioo $(\mathbf{P}<0,05)$.

\section{Infuence du stade de récolte et de l'addition d'urée et de minéraux.}

Les I4 ensilages de plantes entières sans additif ont été ingérés en beaucoup moins grande quantité par le mouton fistulisé que par le lot de moutons utilisé pour les mesures de valeur alimentaire $\left(23,4\right.$ contre $35,3 \mathrm{~g}$ de $\left.\mathrm{MOD} / \mathrm{kg} \mathrm{P}^{\mathbf{0}, 75}\right)$.

Dans le cas de ces I4 ensilages, le pH et la concentration en AGVT du jus de rumen ont été en moyenne respectivement de $6,2 \pm 0,2$ et de $78,2 \pm 7,4 \mathrm{mmoles} / \mathrm{l}$ et ont été liés significativement $(\mathrm{P}<\mathrm{O}, \mathrm{OI})$ l'un à l'autre. Les proportions moyennes d'acides acétique, propionique, butyrique et d'acides ayant une longueur de chaîne supérieure ou égale à 5 ont été respectivement de 53,3 4 4,9 p. I00;27,3 4 4,0 p. IOO ; I6,7 $\pm 2,8$ p. Ioo et $2,7 \pm \mathrm{I}, \mathrm{I}$ p. Ioo. Elles sont donc plus variables dans le cas de la plante ensilée que dans le cas de la plante verte sans que l'on en connaisse les raisons. En effet, que l'on considère le même mais récolté à plusieurs stades ou l'ensemble des ensilages étudiés, ces variations n'ont pas pu être reliées au stade de récolte ou à la composition morphologique et chimique des ensilages. De même, nous n'avons observé aucune liaison entre les principales caractéristiques du jus de rumen et les quantités journalières de matière organique digestible, d'amidon ou de produits préformés dans l'ensilage (acide lactiqute, AGV'T) qui ont été ingérés par le mouton fistulisé. Il en aurait été vraisemblablement de même si l'on avait pris en considération non plus les quantités journalières mais celles ingérées durant les deux heures précédant le prélèvement puisque d'après DULPHY (résultats non publiés), ces deux quantités sont liées significativement dans le cas des ensilages de maïs.

Les 6 ensilages de plantes entières, dans lesquels avait été incorporé à la mise en silo un mélange d'urée et de minéraux, ont été ingérés par les moutons fistulisés en quantité nettement plus importante que les 6 ensilages témoins $(+27$ p. roo) (tab. 3$)$. $\mathrm{L}$ 'incorporation de ce mélange a augmenté significativement $(\mathrm{P}<0, \mathrm{OI})$ la concentration en AGVT du jus de rumen. Elle s'est traduite également par une diminution du $\mathrm{pH}$, faible (de 6, I5 à 5,97), mais significative $(\mathrm{P}<0,05)$. Par contre, elle n'a pas modifié les proportions des différents AGV (tab1. 3).

\section{Ensilages plus ou moins riches en épis}

Lorsque le mouton est passé d'une ration d'ensilage de tiges + feuilles à la ration correspondante d'ensilage de plantes entières, le $\mathrm{pH}$ a diminué de 6,4 à 6,0 et la concentration en AGVT a augmenté de 60,4 à 79,5 mmoles $/ 1$. Au niveau de la composition du mélange, cela s'est traduit par une diminution faible de la proportion d'acide acétique (de 57,2 à 53,2 p. Ioo) au profit de la proportion d'acide propionique qui est passée de 27 , I à 3 I,2 p. Ioo (tab1. 4).

Quand le mouton a reçu non plus une ration d'ensilage de plantes entières mais une ration d'ensilage d'épis (avec les spathes) associé à une quantité limitée de foin 
J. ANDRIEU, C. DEMARQUILIY

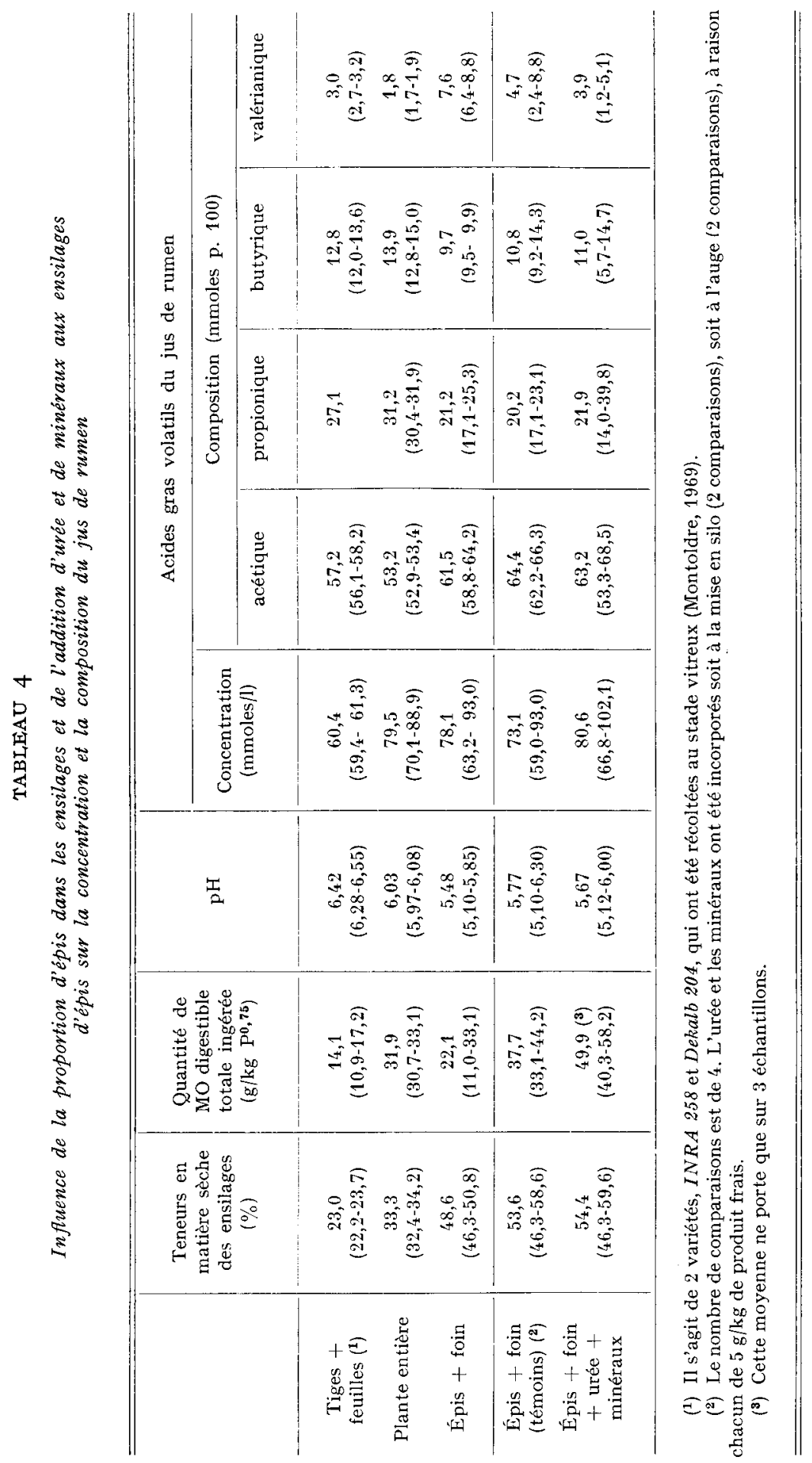


(cette quantité représentait en moyenne I6 p. Ioo de la matière sèche totale ingérée), le $\mathrm{pH}$ a diminué de 6,0 à 5,5 et la concentration du jus de rumen en AGVT n'a pas varié. Par contre, les proportions d'acide acétique et d'acides ayant une longueur de chaîne égale ou supérieure à celle de l'acide valérianique ont augmenté (respectivement de 53,2 à $6 \mathrm{r}, 5 \mathrm{p}$. Ioo et de $\mathrm{I}, 8$ à $7,6 \mathrm{p}$. IOO) au détriment essentiellement de la proportion d'acide propionique (tabl. 4).

L'addition d'urée et de minéraux aux ensilages d'épis n'a entraîné qu'une faible augmentation de la concentration en AGVT du jus de rumen ( + Io p. roo) ; le pH et la composition du mélange d'AGV n'ont pas été modifiés par cet apport.

\section{DISCUSSION}

Les principales caractéristiques du jus de rumen ont été moins variables dans le cas de la plante sur pied que dans celui de la plante ensilée. Cela peut s'expliquer par une variabilité plus importante dans le cas des ensilages, soit au niveau du comportement alimentaire des moutons, soit au niveau de la cinétique des fermentations.

Nous n'avons pas retrouvé les relations mises en évidence par BATH et Rook (I963) entre le niveau d'ingestion de la ration et notamment le $\mathrm{pH}$ et la concentration en $A G V T$ du jus de rumen. Cela est peut être dît au fait que le niveau d'ingestion a beaucoup moins varié dans notre étude.

L'influence du stade de récolte, et donc de la composition de la ration sur les principales caractéristiques du jus de rumen du mouton recevant du maiss sur pied, a été faible. Cependant la concentration en AGVT du jus de rumen a été d'autant plus faible que le stade de récolte était plus tardif, ce qui doit résulter du fait que la vitesse de fermentation des glucides solubles, dont la teneur diminue fortement avec l'âge de la plante au fur et à mesure de leur transformation en amidon dans le grain, est plus élevée que celle de l'amidon. La comıposition du mélange d'AGV du jus de rumen a été très peu influencée par le stade de récolte et donc par les modifications de la composition de la matière organique digestible ingérée (augmentation avec le stade de la proportion d'amidon au détriment des proportions en membranes digestibles et en glucides solubles). Cela peut être dû à deux raisons principales : d'une part, les produits terminaux de la digestion des glucides solubles et de l'amidon dans le rumen sont comparables et d'autre part, la teneur en glucides membranaires de la plante de maìs exploitée entre le début de la formation du grain et le stade vitreux varie relativement peu; c'est ainsi que dans notre étude, la teneur en cellulose brute, qui est un bon critère de la teneur en glucides membranaires, a été en moyenne de I 9,5 土 2,6 p. roo.

La conservation par l'ensilage a peu modifié les caractéristiques du jus de rumen. Elle s'est traduite seulement par une augmentation de la proportion d'acide butyrique au détriment de la proportion d'acide propionique. Cette modification ne peut provenir de l'acide butyrique préformé durant la conservation et apporté par la ration puisque nos ensilages en contenaient peu ou pas. Compte tenu du fait que la conservation s'est traduite essentiellement, au niveau de la composition de la ration, par la substitution de l'acide lactique aux glucides solubles, cette modification de la composition du jus de rumen est peut être due au fait que, dans le rumen, l'acide lactique donne surtout naissance à de l'acide propionique et à de l'acide butyrique 
(WALDo et SCHULTZ, I956 ; WALKER, I968) alors que les glucides solubles semblent donner naissance essentiellement à de l'acide propionique.

L'incorporation d'urée et de minéraux à la mise en silo s'est traduite dans notre étude par une augmentation importante de la concentration en AGVT du jus de rumen, ce qui est en accord avec les travaux de SchAADT et al. (Ig69). Cette augmentation pourrait résulter de l'accroissement de la quantité ingérée, mais ce facteur, nous l'avons vu plus haut, ne semble pas avoir joué. La cause en serait done plutôt, ou surtout, un accroissement de la vitesse de fermentation.

Nos résultats concernant les ensilages de plantes entières sont, en moyenne et dans des conditions de prélèvement identiques, comparables à ceux obtenus par BALWANi et al. (I969) et SchaAdT et al. (1969) sur des moutons et avec des ensilages de maïs. Par contre, ils se différencient nettement de ceux obtenus par CASTLE et WATson (I969-I970) et GRIFFITHS et BATH (I973) sur des bovins et avec des ensilages d'herbe, par des proportions en acide butyrique et surtout en acide propionique plus élevées. Il est possible qu'une différence aussi nette soit due en partie au fait que le type d'animal utilisé pour les mesures n'est pas le même dans les deux cas : en effet, VÉRITE (communication personnelle) a observé sur vaches laitières des différences relativement moins importantes au niveau des orientations fermentaires dans le jus de rumen entre les ensilages d'herbe et de maïs. Par ailleurs, les bovins utilisés dans de telles mesures ont en général à leur disposition un minimum de foin ou de paille (litière) ce qui n'était pas le cas dans nos essais sur moutons. Or, FENNER et al. (I970) ont montré que le fait d'ajouter une quantité limitée de foin (I/3 de la ration) à une ration d'ensilage de maîs entraînait une augmentation de la proportion d'acide acétique dans le jus de rumen. Il semble même qu'avec des régimes à base d'ensilages de maïs l'absence ou la présence de foin joue plus que la teneur en grain, et donc en amidon, de la ration sur la composition du jus de rumen puisque, dans nos essais, la proportion d'acide acétique a augmenté quand les moutons sont passés de l'ensilage de plantes entières distribué seul à l'ensilage d'épi correspondant distribué avec un peu de foin.

Nos résultats montrent que les ensilages de tiges + feuilles récoltés au stade vitreux du grain donnent naissance dans le rumen à une proportion importante d'acide propionique ce qui confirme les résultats de BALwani et al. (I969). D'après ces mêmes auteurs ce serait l'acide lactique, présent en quantité importante dans ces ensilages ( 6 à $8 \mathrm{p}$. Ioo de la matière sèche) qui en serait la cause.

D'un point de vue zootechnique, on peut dire que les rations de mais sur pied ou ensilé semblent particulièrement bien convenir pour l'engraissement car les acides propionique et butyrique qui sont formés en quantité importante avec ce type de ration ont une meilleure efficacité pour l'engraissement que l'acide acétique (ARMsTRONG et BLAXTER, I957). En raison de sa valeur énergétique et de son ingestibilité élevées, 1'ensilage de maïs est aussi très intéressant pour la production laitière. Cependant, le fait qu'il donne naissance à peu d'acide acétique dans le rumen explique pourquoi sa valeur laitière est parfois plus faible que celle des rations à base de fourrages classiques (VÉRITE et JOURNE'T', I97I) : la persistance est en général faible alors que les animaux s'engraissent. Néanmoins, le taux butyreux du lait est élevé par suite de la teneur élevée du jus de rumen en acide butyrique. 


\title{
SUMMARY
}

\author{
COMPOSITION OF RUMEN FLUID \\ IN SHEEP RECEIVING GREEN OR ENSILED MAIZE AD LIBITUM
}

The $\mathrm{pH}$ and concentration of total volatile fatty acids in the rumen fluid of sheep receiving I9 samples of green maize were, on an average, $6.3 \pm 0.1$ and $85.0 \pm 12.3 \mathrm{mMoles} /$ litre of fluid. The molar ( $\mathrm{p}$, Ioo) proportions of acetic, propionic, butyric acids and acids with a chain length corresponding to or exceeding that of valerianic acid were, on an average, $55.2 \pm 3.0 ; 29.6 \pm 3.8$; I3.I \pm 2.6 and $2.1 \pm 0.6$, respectively.

The effect of the growth stage and of its main criteria was inexistent for the $\mathrm{pH}$ and very low for the composition of the mixture of volatile fatty acids, whereas it was high as regards the concentration of total volatile fatty acids : the earlier the growth stage the higher the concentration of total volatile fatty acids.

The amount of digestible organic matter eaten by the sheep only affected the molar proportion of the different volatile fatty acids : the proportion of propionic acid increased to the detriment of the proportion of acetic and butyric acids when the intake of digestible organic matters increased.

The only significant resulting from ensiling the maize was an increase in the proportion of butyric acid to the detriment of the proportion of the propionic acid (table 2 ).

The $\mathrm{pH}$ and concentration of total volatile fatty acids in the rumen fluid of sheep receiving I 4 silages of whole plants without any additive were on an average, $6.2 \pm 0.2$ and $78.2 \pm 7.4$ mMoles/litres, respectively. The molar ( $p$. Ioo) proportions of acetic, propionic, butyric acids and of acids with a chain length corresponding to or exceeding that of valerianic acid were, on an average, $53.3 \pm 4.9 ; 27.3 \pm 4.0 ; 16.7 \pm 2.8$ and $2.7 \pm 1$.I ; respectively.

The characteristics of the rumen fluid from sheep receiving these 14 silages of whole plants did not depend either on the main criteria of the harvest stage or on the amounts of digestible organic matter, starch, or preformed acids in the silage, eaten by the sheep.

The addition of urea and minerals to maïze at ensiling led to a large increase in the amount of organic matter eaten by the sheep and in the concentration of total volatile fatty acids in the rumen fluid. The other characteristics of the rumen fluid were only slightly changed (table 3 ).

The proportions of acetic acid in the rumen fluid were, on an average, $57.2 ; 53.2 ; 61.5$ p. 100 when the sheep received silages of stems + leaves, whole plants and cobs (grain + rachis + husks) from the same maize (table 4 ). However, the maize cob silages were given together with $200 \mathrm{~g}$ hay/day.

The main results are discussed and an attempt is made to draw some conclusions about the animal performances obtained with maize silages.

\section{RÉFÉRENCES BIBLIOGRAPHIQUES}

Andriev J., Demarquilly C., I974a. Valeur alimentaire du maîs fourrage. II. Influence du stade de végétation, de la variété, du peuplement, de l'enrichissement en épis et de l'addition d'urée sur la digestibilité et l'ingestibilité de l'ensilage de maïs. Ann. Zootech., 23, I-25.

Andried J., Demarquilly C., 1974 b. Valeur alimentaire du maïs fourrage. III. Influence de la composition et des caractéristiques fermentaires sur la digestibilité et l'ingestibilité des ensilages de maïs. $A n n$. Zoctech., 23, 27-43.

Armstrong D. G., Blaxter K. L., r957. The utilization of acetic, propionic and butyric acids by fattening sheep. Brit. J. Nutr., 11, 413-425.

Balwani T. L., Johnson R. R., McClure K. E., Dehority B. A., rg69. Evaluation of green chop and ensiled sorghums, corn silage and perennial forages using digestion trials and VFA production in sheep. J. Anim. Sci., 28, 90-97.

Balch D. A., Rowlands S. J., I957. Volatile fatty acids and lactic acid in the rumen of dairy cows receiving a variety of diets. Brit. J. Nutr., 11, 288-298.

BATH I. H., Rook J. A. F., I963. The evaluation of cattle foods and diets in terms of the ruminal concentration of volatile fatty acids. I. The effect of level of intake, frequency of feeding, the ration of hay to concentrates in the diet, and of supplementary feeds. J.Agric. Sci., 61, 34I-348. 
Castle M. E., Watson J. N., I969. Effect of level of protein silage on the intake and production of dairy cows. Br. J. Grassland Soc., 24, I87-194.

Castre M. E., Watson J. N., I97o. Silage and milk production; a comparison between grass silages made with and without formic acid. Br.J. Grassland Soc., 25, 65-7I.

Demarquilly C., 1969. Valeur alimentaire du maïs fourrage. I. Composition chimique et digestibilité du maïs sur pied. Ann. Zootech., 18, 17-32.

Fenner H., Damon R. A., Barnes H. D., I97o. Effects of a stepwise replacement of hay with corn silage on $\mathrm{pH}$, some organic metabolites, and their change in the rumen of the bovine after feeding. J. Dairy Sci, 53, I568-1577.

Griffiths T. W., Bath I. H., I973. Effects of energy and nitrogen supplement of silage diets on rumen fermentation in fistulated heifers. J. A gric. Sci., Camb., 80, 85-89.

Rigaud J., Journet M., r97o. Méthode de dosage des acides gras volatils dans le liquide du rumen. Ann. Biol. anim. Bioch. Biophys., 10, I5I-I57.

SchaddT H., Johnson Jr., R.R., rg69. VFA Production in the rumen of sheep fed limestone and urea treated corn silages. J. Anim. Sci. 29, 839-847.

VÉRIté R., Journet M., I97I. Utilisation comparée de l'ensilage de maïs et de l'ensilage d'herbe pour la production laitière. Ann. Zootech., 20, 153-167.

Waldo D. R., Schultz L. H., I956. Lactic acid production in the rumen. J. Dairy Sci., 39, 1453I 460 .

WALKER D. J., I968. The position of lactic acid and its derivatives on the nutrition and metabolism of ruminants. Nutr., A bstr. Rev., 38, I-I I. 